BNL-114555-2017-IR

CBETA/019

November 2017

\title{
Measurement procedure for CBETA Halbach Magnets
}

\author{
S. Brooks
}

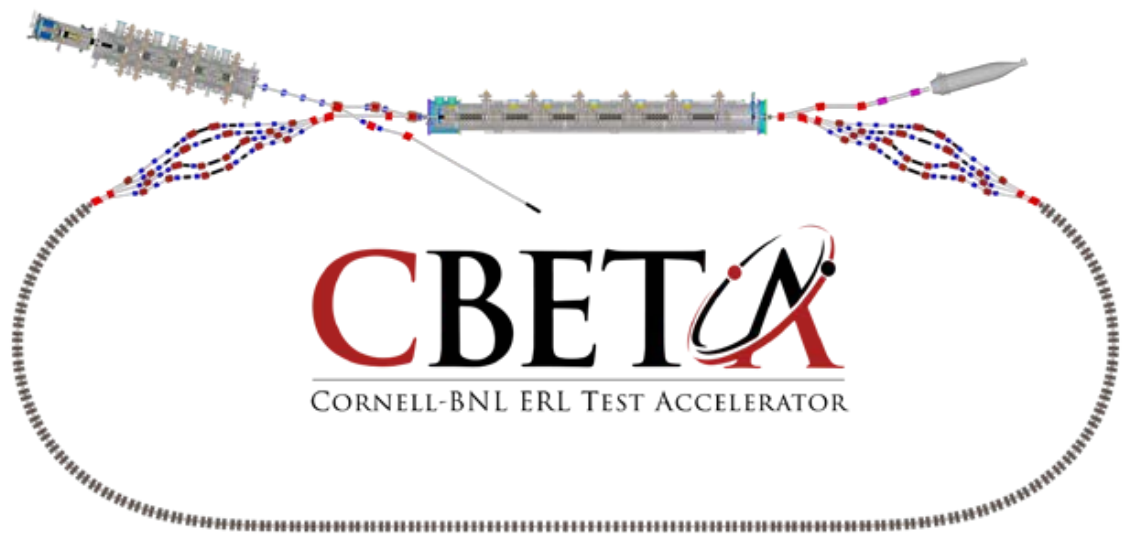

Collider-Accelerator Department, Brookhaven National Laboratory, Upton NY 11973

U.S. Department of Energy

Office of Science, Office of Nuclear Physics

Cornell Laboratory for Accelerator-Based Sciences and Education, Ithaca, NY 14850

Funded by NYSERDA contract 102192

Notice: This document has been authorized by employees of Brookhaven Science Associates, LLC under Contract No. DE-SC0012704 with the U.S. Department of Energy. The United States Government retains a non-exclusive, paid-up, irrevocable, world-wide license to publishor reproduce the published form of this document, or allow others to do so, for United States Government purposes. 


\section{DISCLAIMER}

This report was prepared as an account of work sponsored by an agency of the United States Government. Neither the United States Government nor any agency thereof, nor any of their employees, nor any of their contractors, subcontractors, or their employees, makes any warranty, express or implied, or assumes any legal liability or responsibility for the accuracy, completeness, or any third party's use or the results of such use of any information, apparatus, product, or process disclosed, or represents that its use would not infringe privately owned rights. Reference herein to any specific commercial product, process, or service by trade name, trademark, manufacturer, or otherwise, does not necessarily constitute or imply its endorsement, recommendation, or favoring by the United States Government or any agency thereof or its contractors or subcontractors. The views and opinions of authors expressed herein do not necessarily state or reflect those of the United States Government or any agency thereof. 


\section{Measurement Procedure for CBETA Halbach Magnets}

Stephen Brooks 2017-Nov-08 CBETA machine note \#019

\section{Pre-requisites}

\subsection{Magnet Orientation}

The CBETA magnets will be marked by the manufacturer with two sides called " $\mathrm{A}$ " and " $\mathrm{B}$ ", as well as which part of the magnet is the "top" vertically. The significances of these sides are given in the table below.

\section{Side A}

Beam-downstream (DS)

Faces the rotating coil motor

The "front" in any 2D magnet design drawings

\section{Side B}

\section{Beam-upstream (US)}

Faces away from the rotating coil motor

\subsection{Magnet Measurement}

In all cases, magnets will be measured:

- With water cooling attached, at the equilibrium temperature of $85 \mathrm{~F}$, which may be achieved by running the chiller through the magnet for at least 40 minutes $^{1}$ before testing;

- With a vertical dipole window-frame corrector surrounding the Halbach core.

\subsection{Magnet Survey}

The following must be included in the survey measurements:

- The roughly 8 magnet fiducial points, located on the front and back faces of the aluminium body of each magnet;

- At least 3 points on the rotating coil base plate, to define the "bench frame";

- An accurate point set on the rotating coil cylinder relative to the base plate, which enables reconstruction of the coil axis.

Optionally, the magnet longitudinal location can be better determined by surveying:

- Points on the exposed surface of the 16 permanent magnet wedges, on both the $A$ and $B$ sides of the magnet.

For the rotating coil, and accurate survey method is to take sets of points on the surface of the coil either side of the magnet, at multiple orientations as the coil rotates about its axis. This is expected

\footnotetext{
${ }^{1}$ Magnet warm-up is dominated by BNL chiller power limitations. Observed cool-down times are 20-30 minutes, although this could vary with ambient temperature, hence a safety margin has been added to this wait time.
} 
to be accurate and repeatable relative to the base plate to 20 microns or better, but needs checking. If repeatability is confirmed, this detailed coil survey measurement could be done less often.

All magnet surveys must be treated as independent measurements of different sets of points, with neither "pre-survey" of the magnet nor fitting to an assumed model of the magnet. The base plate fiducials and latest accurate coil position should always be included in the survey model.

\section{Rotating Coil Roll Angle Calibration}

To determine the roll angle of the coil itself, a magnet must be measured and surveyed twice in the forward and reversed orientations. This will be done at the start of the CBETA measurements and also whenever a significant roll angle change is observed in the daily readings of the vertical corrector dipole (described in the next section).

1. Measure the field direction of the vertical dipole window-frame corrector using the rotating coil and the corrector's power supply

2. Ensure the window-frame corrector power is switched off

3. Retract rotating coil

4. Insert Halbach core into window-frame corrector

5. Insert rotating coil

6. In parallel, starting from now, survey can take measurements of magnet fiducials relative to coil and bench

7. Switch on chiller and wait for thermal equilibration time of Halbach core

8. Take a rotating coil reading (about 20 records)

9. Retract rotating coil

10. Rotate the Halbach core 180 degrees about vertical axis

11. Perform steps 5 through 8 above again, producing a second coil reading and a second set of survey fiducials for the reversed magnet

\section{Daily Vertical Corrector Dipole Measurement}

Measure the vertical corrector dipole's direction relative to the coil at the beginning and end of each day (on each coil bench).

1. Remove any Halbach core from the window-frame

2. Measure the field direction of the vertical dipole window-frame corrector using the rotating coil and the corrector's power supply

3. Ensure the window-frame corrector power is switched off for subsequent measurements of Halbach cores

If the vertical corrector dipole angle relative to the coil has changed significantly, e.g. by more than 1 mrad (which is half the CBETA roll angle tolerance) or any unusually large change, the rotating coil roll angle calibration should be repeated using the reversed magnet (previous section). Additionally, any magnet measurements made during the period when the angle change took place should be repeated. 


\section{First Measurement of Magnet (without Tuning Rods)}

This measurement does not need to include survey measurements. It is mainly to determine the required tuning rod arrangement (or to see if there is a major error that is not tuneable).

1. Insert Halbach core into window-frame corrector

2. Switch on chiller and wait for thermal equilibration time of Halbach core

3. Take a rotating coil reading (about 20 records)

4. Calculate tuning rod arrangement using the PM_Correct software from Stephen Brooks, using the rotating coil ".dat" file as input

5. Fill two tuning rod cartridges with wires of appropriate lengths (may be automated)

6. Ensure the rods are glued securely in place and will not move when placed into magnet

\section{Magnet Measurement after Tuning}

The first and any subsequent tuning iterations will also be surveyed because they have a significant likelihood of being of acceptable field quality, so the survey data of each magnet needs to be available.

1. If not already there, insert Halbach core into window-frame corrector

2. Insert tuning rod cartridges for this iteration, ensuring the marker " $A L$ " is on side $A$ of the magnet and on the left-hand side as seen from side $A$, and the marker " $A R$ " is on side $A$ on the right-hand side

3. In parallel, starting from now, survey can take measurements of magnet fiducials relative to coil and bench

4. If magnet is not already at thermal equilibrium, switch on chiller and wait for thermal equilibration time

5. Take a rotating coil reading (about 20 records)

6. The PM_Correct software from Stephen Brooks will verify if the tuned magnet meets CBETA figures of merit and if necessary will suggest a further optional tuning rod iteration, using the rotating coil ".dat" file as input

\section{Software Packages}

There will be three software packages provided by Stephen Brooks for use with CBETA magnet measurement: PM_Correct, PM_Survey and PM_CoilRoll.

\subsection{Field Quality and Tuning Rod Calculation (PM_Correct)}

This takes the rotating coil ".dat" file as an input and calculates:

- Multipole harmonic coefficients relative to the coil axis, including integrated dipole and quadrupole;

- Re-centered multipole coefficients where the magnet displacement and orientation are fitted to give the nominal dipole field at the centre and normal quadrupole orientation;

- The displacement and rotation relative to the coil axis required to give the best fit above;

- Field quality figures of merit based on the above multipole coefficients and limits derived from CBETA-specific tracking studies; 
- The lengths of tuning wire that must be inserted into each slot of the wire holder in order to correct the observed multipole and strength errors.

This program may also control an automated wire cutter robot to help with filling the tuning rod cartridges.

This program will also generate output log files in CSV (comma-separated) or LaTeX (hence PDF) formats, which will be saved with systematic filenames on a network drive.

\subsection{Magnet Survey: Magnet Axis to Global Coordinates (PM_Survey)}

This takes as input:

- Survey file of magnet fiducials and rotating coil axis in bench frame;

- Displacements of the measured ideal magnet axis from the rotating coil position;

- Roll angle of the ideal magnet orientation relative to the rotating coil zero;

- BMAD-generated survey file of ideal magnet axes in CBETA global coordinates.

This program outputs the positions that the magnet fiducials should have in CBETA global coordinates when installed. These positions will place the measured ideal magnet axis on the ideal magnet axis specified by BMAD.

\subsection{Rotating Coil Roll Angle Calibration (PM_CoillRoll)}

This takes as input:

- Two survey files of magnet fiducials and rotating coil axis in bench frame, one in normal orientation and one reversed;

- Two rotating coil measurements corresponding to the above surveys.

This program computes the ideal magnetic axis in both forward and backward orientations and uses the redundant information to calculate the roll angle of the coil itself (relative to the bench) as a best fit using the survey points and orientation of multipoles measured.

Once the coil roll angle is known, this may be input to the single-measurement magnet survey program and subsequent magnets will only require one measurement for as long as the coil roll angle is assumed to be fixed.

\section{Mapping to 4-Digit Identifiers in Magnet Division Database}

The BNL magnet division uses 4-digit numbers to identify distinct magnets. Various codes under "ERHIC-PMQ" for permanent magnets have already been used:

\begin{tabular}{|l|l|l|}
\hline Numerical Range & Count & Magnet Types \\
\hline $00 \mathrm{nn}, 01 \mathrm{nn}, 02 \mathrm{nn}$ & $?$ & Early R\&D permanent magnets \\
\hline $0301-0306$ & 6 & ATF-FFAG BD magnets \\
\hline $04 \mathrm{nn}$ & $?$ & CBETA iron hybrid prototype magnets \\
\hline $0501-0506$ & 6 & ATF-FFAG QF magnets \\
\hline $1301-1304$ & 4 & CBETA first girder BD magnets \\
\hline $1501-1504$ & 4 & CBETA first girder QF magnets \\
\hline
\end{tabular}


The suggested numerical ranges for the full CBETA magnet quantities are shown in the table below. Note that the manufacturer will place a serial number on the magnets, counted within each magnet type, so for example QF serial number $\mathrm{N}$ will be given identifier $2500+\mathrm{N}$.

\begin{tabular}{|l|l|l|}
\hline Numerical Range & Count & Magnet Types \\
\hline $2101-21 \mathrm{nn}$ & $\geq 28$ & CBETA production BDT1 magnets \\
\hline $2201-22 \mathrm{nn}$ & $\geq 22$ & CBETA production BDT2 magnets \\
\hline $2301-23 \mathrm{nn}$ & $\geq 30$ & CBETA production BD magnets \\
\hline $2401-24 \mathrm{nn}$ & $\geq 27$ & CBETA production QD magnets \\
\hline $2501-26 \mathrm{nn}$ & $\geq 107$ & CBETA production QF magnets \\
\hline $2701-270 \mathrm{n}$ & $2(?)$ & Halbach magnets for splitter lines S4, R4 \\
\hline 2731 & 1 & BDH (half length BD) magnet \\
\hline 2751 & 1 & QFH (half length QF) magnet \\
\hline
\end{tabular}

If some magnets are broken and extra magnets have to be built, the number of magnet identifiers may exceed the number of magnets installed in the machine. The $27 \mathrm{nn}$ range is used for various "orphan" magnet types being built at BNL. 